\title{
Research on model-based calculation of greenhouse gas emissions from domestic wastewater treatment systems in Vietnam
}

\author{
Nghiên cứu tính toán phát thải khí nhà kinh tù hệ thống xử lý nước thải sinh \\ hoạt dựa trên mô hìh tại Việt Nam \\ Research article
}

Nguyen, Thi Van Anh ${ }^{1} *$; Dang, Xuan Hien ${ }^{1}$; Nguyen, Duc Toan ${ }^{2}$

${ }^{1}$ Institute of Science and Environmental Technology, Hanoi University of Science and Technology (HUST), No1 Dai Co Viet, Hai Ba Trung, Hanoi, Vietnam; ${ }^{2}$ Agency of Seas and Islands use management, Vietnam Administration of Seas and Islands (VASI), No 83 Nguyen Chi Thanh, Dong Da, Hanoi, Vietnam

\begin{abstract}
There are three important greenhouse gases: carbon dioxide $\left(\mathrm{CO}_{2}\right)$, methane $\left(\mathrm{CH}_{4}\right)$ and nitrous oxide $\left(\mathrm{N}_{2} \mathrm{O}\right)$, which are generated from the domestic wastewater treatment systems, including on-site and off-site sources. On-site emission of greenhouse gases occurs during process of wastewater treatment, while the off-site emission of greenhouse gases occurs during energy using and other supporting activities of the treatment system. The research established model to calculate greenhouse gas emissions from the domestic wastewater treatment systems, was named No.0 MTH model. The No.0 MTH model was based on balance equations of substrate and biomass, biochemical reactions and Monod kinetics equations for biological treatment reactors and writen by programing Scalable language. Model was calibrated and applied on the Yen So wastewater treatment plant, Ha Noi and the results were obtained at $22^{\circ} \mathrm{C}$ as follows: off-site $\mathrm{GHG}$ emission is $29,560 \mathrm{kgCO}_{2}$-eq/day; on-site GHG emission is $13,534 \mathrm{kgCO}_{2}$-eq/day, and the rate of on-site emission is $2.506 \mathrm{kgCO}_{2}$-eq/ $\mathrm{kg}$ BOD. Maybe using the No.0 MTH model to calculate greenhouse gas emissions from the domestic wastewater treatment systems with similar biological methods.
\end{abstract}

Có 3 khí nhà kính quan trọng là khí Cacbonic $\left(\mathrm{CO}_{2}\right)$, khí Mêtan $\left(\mathrm{CH}_{4}\right)$, và khí Đinito monoxit $\left(\mathrm{N}_{2} \mathrm{O}\right)$ được phát sinh tù hệ thống xủ lý nước thải sinh hoạt gồm cả nguồn trực tiếp và gián tiếp. Phát thải trục tiếp khi nhà kính (KHK) xảy ra trong suốt quá trình xủ lý còn phát thải gián tiếp khí nhà kính xảy ra trong quá trình sủ dụng năng luợng và các hoạt động phụ trợ bên ngoài hệ thống xủ lý. Nghiên cưu đã thiết lập mô hình tính toán phát thải khí nhà kính tù hệ thống xử lý nước thải sinh hoạt, được đặt tên là mô hình MTH số 0 . Mô hình MTH số 0 đã dựa trên các phương trình cân bằng khối lương co chất và sinh khối, các phản ứng hóa sinh và phưong trình Monod đối với các thiết bị xư lý sinh học và được viết trên ngôn ngũ lập trình scala. Mô hình đã được hiệu chỉnh và được áp dụng tính toán tại nhà máy xủ lý nuoớc thải sinh hoạt Yên Sở, thành phố Hà Nội và kết quả thu được tại $22^{\circ} \mathrm{C}$ nhu sau: phát thải $\mathrm{KNK}$ gián tiếp là $29.560 \mathrm{kgCO}_{2-t d}$ ngày và phát thải $\mathrm{KNK}$ trục tiếp là $13.534 \mathrm{kgCO}$-tđđ/ngày với tỷ lẹ phát thải khí nhà kính trục tiếp là 2,506 $\mathrm{kgCO}$-tol $\mathrm{kgBOD}$. Có thể sủ dụng mô hình MTH số 0 để tính toán phát thải khí nhà kính tù hệ thống xủ̉ lý nước thải sinh hoạt bằng phưong pháp sinh học tương tụ.

Keywords: greenhouse gas, on-site and off-site emissions, domestic wastewater treatment plant

\section{Introduction}

Climate change has been more and more complicated and serious, so the decrease in greenhouse gases (GHGs) emission plays an important role. The emission of GHGs from wastewater treatment systems is the problem of interest and can be measured and assessed to determine the sustainability of treatment systems. There are three main GHGs: carbon dioxide $\left(\mathrm{CO}_{2}\right)$, methane $\left(\mathrm{CH}_{4}\right)$ and nitrous oxide $\left(\mathrm{N}_{2} \mathrm{O}\right)$, which are generated from the domestic wastewater treatment systems, including on-site and off-site sources. 
The off-site GHGs emission includes electricity production; production and transportation of fuel and materials; landfill treatment of the generated solid waste sludge from the on-site processes. The major on-site sources of GHGs generation are aerobic and anaerobic bioreactors, anaerobic digester, biogas leakage, chemical coagulation/flocculation process, and biogas combustion in recovery boilers. The aim of research was to establish the No.0 MTH model to estimate the GHGs emission from wastewater treatment systems. The model was based on balance equations of substrate, biomass, biochemical reactions and Monod kinetics equations for biological treatment reactors; the hypothesis was that during wastewater treatment process, $\mathrm{C}_{10} \mathrm{H}_{19} \mathrm{NO}_{3}$ and $\mathrm{C}_{5} \mathrm{H}_{7} \mathrm{NO}_{2}$ were used to represent the substrate and the biomass, respectively. The model was solved by numerical method and then was coded. It was obvious that the model could be significantly useful for assessment of domestic wastewater treatment systems in Vietnam, in order to increase the efficiency of wastewater treatment systems for sustainable development and deal with climate change.

\section{Materials and methods}

\subsection{Research objective}

The Yen So domestic wastewater treatment plant, Ha Noi was used to calculate the greenhouse gases (GHGs) emission.

\subsection{Theoretical basis for the development of No. 0 MTH model}

2.2.1. Estimation of the off-site greenhouse gas emissions

The main off-site sources of greenhouse gas emission include electricity production for on-site use, production and transportation of fuel and materials. The overall off-site emission of greenhouse gases is obtained by addition of the produced gases by each source.

$$
\begin{aligned}
& \mathrm{P}_{\mathrm{CO} 2 \text {, off-site }}=\mathrm{P}_{\mathrm{CO} 2 \text {, electricity }}+\mathrm{P}_{\mathrm{CO} 2 \text {, natural gas }}+\mathrm{P}_{\mathrm{CO} 2 \text {, material }} \\
& +\mathrm{P}_{\mathrm{CO} 2 \text {, electricity }}=\mathrm{QE} * \sum\left(P F_{i} * \mathrm{EF}_{i}\right)
\end{aligned}
$$

where:

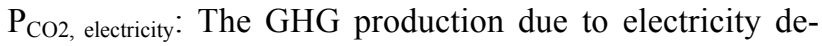
mands of the plant $\left(\mathrm{kgCO}_{2 \text {-td }} /\right.$ day)

QE: The quantity of electricity used for the operation of the entire plant (kwh/day)

$\mathrm{PF}_{\mathrm{i}}$ : Percentage contribution of fuel i to satisfy electricity generation needs of the wastewater treatment systems (\%) (Table 1)

$\mathrm{EF}_{\mathrm{i}}$ : GHGs emission factor of fuel $\mathrm{i}$ in producing GHGs $\left(\mathrm{kgCO}_{2 \text {-td }} / \mathrm{kwh}\right)$ (Table 2-1)

$+\mathrm{P}_{\mathrm{CO} 2 \text {, natural gas }}=\left[\mathrm{Q}_{\text {natural gas }} * \mathrm{EF}_{\text {natural gas, } \mathrm{CO} 2} / 10^{3}\right]+25 *[\mathrm{Q}$ natural gas $\left.* \mathrm{EF}_{\text {natural gas, } \mathrm{CH} 4} / 10^{3}\right]+296 *\left[\mathrm{Q}_{\text {natural gas }} * \mathrm{EF}_{\text {natural }}\right.$ gas, N2O $\left./ 10^{3}\right] \quad(2-3)$

where:

$\mathrm{P}_{\mathrm{CO} 2 \text {, natural gas }}$ : The off-site GHGs production because of natural gas consumption for space heating in the plant $\left(\mathrm{kgCO}_{2-}\right.$ td/day)

$\mathrm{Q}_{\text {natural gas: }}$ The quantity of natural gas used for space heating in the plant $\left(\mathrm{m}^{3} /\right.$ day)

$\mathrm{EF}_{\text {natural gas, } \mathrm{CO} 2}, \mathrm{EF}_{\text {natural gas, } \mathrm{CH} 4}, \mathrm{EF}_{\text {natural gas, } \mathrm{N} 2 \mathrm{O} \text { : the overall }}$ natural gas $\mathrm{CO}_{2}, \mathrm{CH}_{4}$ and $\mathrm{N}_{2} \mathrm{O}$ emissions factors $\left(\mathrm{g} / \mathrm{m}^{3}\right)$ (Table 2)

$+P_{\mathrm{CO}_{2} \text {,material }}=\sum_{i} Q_{\text {material }}^{i} \mathrm{EF}_{\text {materiali }}$

where:

$\mathrm{P}_{\mathrm{CO} 2 \text {, material: }}$ The off-site GHGs production because of material consumption in the plant $\left(\mathrm{kgCO}_{2 \text {-td }} /\right.$ day)

$\mathrm{Q}_{\text {material }}^{\mathrm{i}}$ : The quantity of material used in the plant $(\mathrm{kg} /$ day) $\mathrm{EF}^{\mathrm{i}}$ material: The emission factor of material $\mathrm{i}$ in the plant $\left(\mathrm{kgCO}_{2 \text {-td }} / \mathrm{kg}\right.$ material) (Table 3)

+ The off-site greenhouse gases emission factors are in the below tables:

Table 1. Emission factors for different methods of electricity production $[1,2,4]$

\begin{tabular}{lccccc} 
Fuel types & Hydro & Nuclear & Coal & Other fuel & Bio-energy, wind, tidal \\
\hline $\mathrm{EF}_{\mathrm{i}}\left(\mathrm{gCO}_{2-\mathrm{eq}} / \mathrm{kwh}\right)$ & 10 & 9 & 877 & 604 & 11 \\
$\mathrm{PF}_{\mathrm{i}}(\%)$ & 48.78 & - & 23.07 & 27.72 & 0.43
\end{tabular}

Table 2. Emission factorsfrom production and transportation of fuel $\left(\mathrm{g} / \mathrm{m}^{3}\right)$ [3]

\begin{tabular}{ccc}
$\mathrm{EF}_{\text {natual gas, } \mathrm{CO} 2}$ & $\mathrm{EF}_{\text {natual gas, } \mathrm{CH} 4}$ & $\mathrm{EF}_{\text {natual gas, } \mathrm{N} 2 \mathrm{O}}$ \\
\hline 431.9 & 2.1 & 0.000022
\end{tabular}

Table 3. Emission factors of materials $\left(\mathrm{kgCO}_{2-\mathrm{eq}} / \mathrm{kg} \mathrm{ma-}\right.$ terial) [2]

\begin{tabular}{lccc} 
Material types & Methanol & Alkalinity & $\mathrm{FeCl}_{3} .6 \mathrm{H}_{2} \mathrm{O}$ \\
\hline $\mathrm{EF}_{\text {material }}$ & 1.54 & 1.74 & 2.71
\end{tabular}

2.2.2. Estimation of the on-site greenhouse gases emissions

\section{a. Calculation in the first boundary}

The removal of BOD, suspended solid (SS) in the primary clarifier:

$\mathrm{BOD}_{\text {removal,clarifier }}=\operatorname{Pr}_{\text {clarifier,BOD }} \times \mathrm{Q}_{\mathrm{o}, \mathrm{v}} \times \mathrm{S}_{\mathrm{o}, \mathrm{v}}$
$\mathrm{SS}_{\text {removal,clarifier }}=\operatorname{Pr}_{\text {clarifier,SS }} \times \mathrm{Q}_{\mathrm{o}, \mathrm{v}} \times \mathrm{X}_{\mathrm{o}, \mathrm{v}}$

where:

$\mathrm{BOD}_{\text {removal,clarifier }}$; $\mathrm{SS}_{\text {removal,clarifier: the amount of BOD and }}$ $\mathrm{SS}$ removal in the primary sedimentation tank, respectively (g/day)

$\mathrm{Pr}_{\text {clarifier,BOD}}$; $\mathrm{Pr}_{\text {clarifier,Ss: }}$ the percentage of $\mathrm{BOD}_{5}$ and SS removal in the primary sedimentation tank, respectively (\%) $\mathrm{Q}_{\mathrm{o}, \mathrm{v}}$ : influent wastewater flow rate $\left(\mathrm{m}^{3} /\right.$ day $)$ 
$\mathrm{S}_{\mathrm{o}, \mathrm{v}}, \mathrm{X}_{\mathrm{o}, \mathrm{v}}$ : influent substrate and suspended solids concentration, respectively $(\mathrm{mg} / \mathrm{l})$

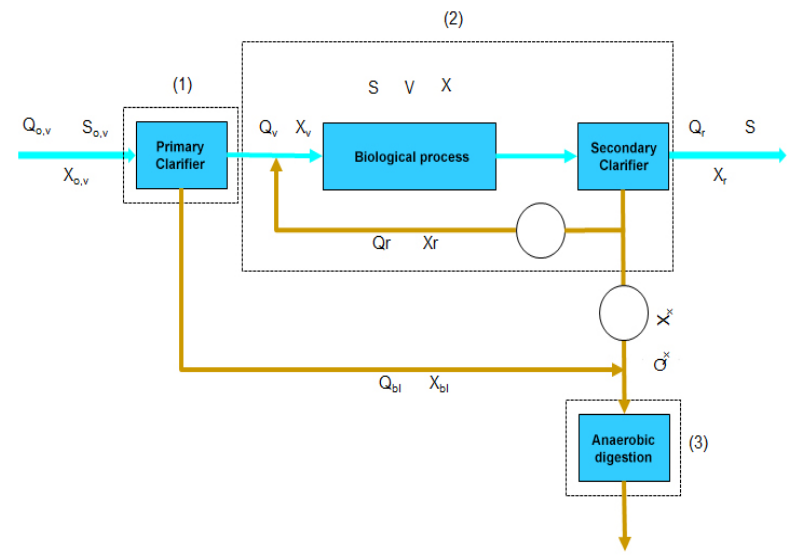

Figure 1. Flowchart of the wastewater treatment system

\section{b. Calculation in the second boundary}

The balance equations for substrate and biomass concentrations in the aerobic bioreactor as follows:

$V \cdot \frac{d S}{d t}=Q_{v} \cdot S_{v}-\left(Q_{r} \cdot S_{r}+Q_{x} \cdot S_{x}\right)+r_{s} \cdot V$

$V \cdot \frac{d X}{d t}=Q_{v} \cdot X_{v}-\left(Q_{r} \cdot X_{r}+Q_{x} \cdot X_{x}\right)+r_{x} \cdot V$

$r_{s}=-\frac{k \cdot X \cdot S}{K_{s}+S}$

$r_{x}=-Y . r_{s}-k_{d} \cdot X$

where:

$\mathrm{V}$ : The volume of the aerobic bioreactor $\left(\mathrm{m}^{3}\right)$

$(d X) ;(d S)$ : the change rate of biomass and substrate con-

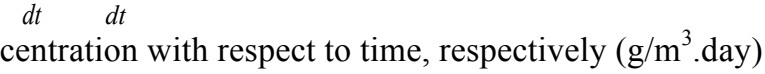

$\mathrm{X}, \mathrm{S}$ : Substrate and biomass concentrations inside the aerobic bioreactor, respectively $(\mathrm{mg} / \mathrm{l})$

$\mathrm{Q}_{\mathrm{v}}, \mathrm{Q}_{\mathrm{r}}, \mathrm{Q}_{\mathrm{x}}$ : Inflow wastewater rate, outflow wastewater rate and discharge of sludge flow in the aeration tank, respectively $\left(\mathrm{m}^{3} /\right.$ day $)$

$\mathrm{X}_{\mathrm{r}}, \mathrm{S}_{\mathrm{r}}$ : The concentration of effluent biomass and substrate, respectively $(\mathrm{mg} / \mathrm{l})$

$\mathrm{r}_{\mathrm{x}}$ : Net rate of biomass production in the aeration tank $\left(\mathrm{g} / \mathrm{m}^{3}\right.$.day)

$\mathrm{r}_{\mathrm{s}}$ : Substrate utilization rate in the aeration tank $\left(\mathrm{g} / \mathrm{m}^{3}\right.$.day) In the steady-state condition, $\frac{d S}{d t}=0$ and $\frac{d X}{d t}=0$ and using $\frac{1}{S R T}=\frac{Y k S}{K S+S}-k_{d}$ and $H T R=\frac{V}{Q_{v}}$

The substrate and biomass concentrations inside the aerobic bioreactor are obtained as:

$$
\begin{aligned}
& S=\frac{K_{s}\left[1+\mathrm{k}_{d} \cdot \mathrm{SRT}\right]}{S R T .\left(Y k-k_{d}\right)-1} \\
& X=\left(\frac{S R T}{H R T}\right)\left\{\frac{Y \cdot\left(S_{v}-S\right)}{1+k_{d} \cdot S R T}\right\}
\end{aligned}
$$

The total suspended solid (SS) in the system could be obtained as:

$\mathrm{X}_{\text {Total }, \mathrm{SS}}=\mathrm{X}+\mathrm{X}_{\mathrm{nb}}+\mathrm{X}_{\mathrm{nit}}$

$$
\begin{aligned}
& X_{n b}=f_{d} \cdot k_{d} \cdot X \cdot S R T+\frac{X_{n b, v} \cdot S R T}{H R T} \\
& \text { celldebris } \\
& \text { non-biodeg radableVSS } \\
& X_{n b, v}=V S S .\left(1-\frac{b p C O D}{p C O D}\right) \\
& X_{n i t}=\left(\frac{S R T_{n i t}}{H R T}\right)\left\{\frac{Y_{n i t} \cdot N}{1+k_{d, n i t} \cdot S R T_{n i t}}\right\} \\
& P_{S S}=\frac{X_{\text {Total }, S S} \cdot V}{S R T}=P_{S S, B O D}+P_{S S, \text { nit }}+P_{S S, \text { celldebris }}+P_{S S, n b V S S} \\
& P_{S S, n i t}=\frac{X_{\text {nit }} \cdot V}{S R T} \quad(2-18) ; P_{S S, B O D}=\frac{X . V}{S R T} \\
& P_{S S, \text { celldebris }}=f_{d} \cdot k_{d} \cdot X \cdot V \\
& \mathrm{P}_{S S, n b V S S}=\mathrm{Q}_{\mathrm{v}} \cdot \mathrm{X}_{\mathrm{nb}, \mathrm{VSS}} \\
& B O D_{\mathrm{OX}}=Q_{v}\left(S_{v}-S\right)-r_{O 2, \text { decay }} \cdot\left(P_{S S}-Q_{v} \cdot X_{n b, v}\right) \\
& \mathrm{VSS}_{\text {decay }}=0,85 \cdot \mathrm{V} \cdot\left(\mathrm{k}_{\mathrm{d}} \cdot \mathrm{X}+\mathrm{k}_{\mathrm{d}, \mathrm{nit}} \cdot \mathrm{X}_{\text {nit }}\right) \\
& \mathrm{N}_{2} \mathrm{O}=Q_{v} \cdot T K N_{v} \cdot R_{\mathrm{N}_{2} \mathrm{O}} \\
& \mathrm{CO}_{2, \text { BODremoval }}=\mathrm{Y}_{\mathrm{CO} 2} \cdot\left(\mathrm{BOD}_{\mathrm{OX}}-\mathrm{BOD}_{\mathrm{OX}, \mathrm{dnt}}\right) \\
& \mathrm{CO}_{2, \text { VSSdecay }}=Y_{\mathrm{CO}_{2}, \text { decay }} . V S S_{\text {decay }} \\
& \mathrm{CO}_{2, \mathrm{dnt}}=\mathrm{Y}_{\mathrm{CO} 2, \mathrm{dnt}} \cdot \mathrm{N} \cdot \mathrm{Q}_{\mathrm{v}} \\
& \mathrm{CO}_{2 \text {,consumption nit }}=\mathrm{r}_{\mathrm{CO} 2 \text {,nit }} \text { N. } Q_{\mathrm{v}}
\end{aligned}
$$

The total amount of $\mathrm{CO}_{2}$ for the aerobic process can be calculated as follow:

$\begin{array}{lc}\mathrm{CO}_{2, \text { production in aerobic process }}=\mathrm{CO}_{2, \mathrm{BOD} \text { removal }}+\mathrm{CO}_{2, \mathrm{VSS} \text { decay }}+ \\ \mathrm{CO}_{2, \text { dnt }}-\mathrm{CO}_{2, \text { consumption nit }} & (2-29) \\ \mathrm{CO}_{2, N_{2} \text { Oemission }}=296 * \mathrm{~N}_{2} \mathrm{O}_{\text {nitrogenremoval }} & (2-30)\end{array}$

\section{c. Calculation in the third boundary}

The total amount of $\mathrm{CO}_{2}$ and $\mathrm{CH}_{4}$ production in the anaerobic digester could be calculated as follows:

$$
\begin{aligned}
& C O_{2, \text { digester }}=Y_{\mathrm{CO}_{2}, d r} \cdot B O D_{\text {removal,dr }}+Y_{\mathrm{CO}_{2}, \text { decay }}^{d r} \cdot V S S_{\text {decay }}^{d r} \\
& \mathrm{CH}_{4, \text { digester }}=Y_{\mathrm{CH}_{4}, \mathrm{dr}} \cdot \mathrm{BOD}_{\text {removal }, d r}+Y_{\mathrm{CH}_{4}, \text { decay }}^{d r} \cdot V S S_{\text {decay }}^{d r} \\
& \mathrm{CO}_{2, \text { digestermethane }}=Y_{\mathrm{CH}_{4} \text {, combustion }} . \mathrm{CH}_{4, \text { digesterrecovery }}+23 .\left(\mathrm{CH}_{4, \text { digesterdissolve }}\right. \\
& \left.+\mathrm{CH}_{4, \text { digesterleak }}\right)
\end{aligned}
$$

\section{d. Establishing of emission factors of greenhouse gases in wastewater treatment system}

In domestic wastewater, $\mathrm{C}_{10} \mathrm{H}_{19} \mathrm{NO}_{3}$ and $\mathrm{C}_{5} \mathrm{H}_{7} \mathrm{NO}_{2}$ were used to represent the substrate and the biomass, respectively. The chemical reactions in aerobic bioreactor: reactions of biomass decay, nitrification and denitrification as well as the anaerobic decays are as follows:

$$
\begin{aligned}
& 0,02 \mathrm{C}_{10} \mathrm{H}_{19} \mathrm{NO}_{3}+0,01 \mathrm{NH}_{4}^{+}+0,01 \mathrm{HCO}_{3}^{-}+0,1 \mathrm{O}_{2} \\
& \rightarrow 0,03 \mathrm{C}_{5} \mathrm{H}_{7} \mathrm{NO}_{2}+0,11 \mathrm{H}_{2} \mathrm{O}+0,06 \mathrm{CO}_{2} \\
& 0,05 \mathrm{C}_{5} \mathrm{H}_{7} \mathrm{O}_{2} \mathrm{~N}+0,25 \mathrm{O}_{2} \rightarrow 0,2 \mathrm{CO}_{2}+0,05 \mathrm{NH}_{4}^{+} \\
& +0,05 \mathrm{HCO}_{3}^{-}+0,05 \mathrm{H}_{2} \mathrm{O} \\
& 0,02 \mathrm{C}_{10} \mathrm{H}_{19} \mathrm{NO}_{3}+0,1 \mathrm{H}_{2} \mathrm{O} \rightarrow 0,118 \mathrm{CH}_{4}+0,052 \mathrm{CO}_{2} \\
& +0,0025 \mathrm{C}_{5} \mathrm{H}_{7} \mathrm{O}_{2} \mathrm{~N}+0,0175 \mathrm{NH}_{4}^{+}+0,0175 \mathrm{HCO}_{3}^{-} \\
& 0,1275 \mathrm{NH}_{4}^{+}+0,24 \mathrm{O}_{2}+0,01 \mathrm{CO}_{2}+0,0025 \mathrm{HCO}_{3}^{-} \rightarrow \\
& 0,0025 \mathrm{C}_{5} \mathrm{H}_{7} \mathrm{O}_{2} \mathrm{~N}+0,125 \mathrm{NO}_{3}^{-}+0,25 \mathrm{H}^{+}+0,1225 \mathrm{H}_{2} \mathrm{O}
\end{aligned}
$$




$$
\begin{aligned}
& 0,02 \mathrm{C}_{10} \mathrm{H}_{19} \mathrm{NO}_{3}+0,184 \mathrm{NO}_{3}^{-}+0,184 \mathrm{H}^{+} \rightarrow 0,004 \mathrm{C}_{5} \mathrm{H}_{7} \mathrm{O}_{2} \mathrm{~N} \\
& +0,02 \mathrm{NH}_{4}^{+}+0,02 \mathrm{HCO}_{3}^{-}+0,09 \mathrm{~N}_{2}+0,162 \mathrm{CO}_{2}+0,22 \mathrm{H}_{2} \mathrm{O} \\
& 0,02 \mathrm{C}_{10} \mathrm{H}_{19} \mathrm{NO}_{3}+0,18 \mathrm{H}_{2} \mathrm{O} \rightarrow 0,003 \mathrm{C}_{5} \mathrm{H}_{7} \mathrm{O}_{2} \mathrm{~N}+0,118 \mathrm{CH}_{4} \\
& +0,052 \mathrm{CO}_{2}+0,017 \mathrm{NH}_{4}^{+}+0,017 \mathrm{HCO}_{3}^{-}
\end{aligned}
$$

\begin{tabular}{|c|c|c|}
\hline \multicolumn{3}{|c|}{ Aerobic process } \\
\hline Parameter & Unit & Value \\
\hline $\mathrm{Y}_{\mathrm{CO} 2}$ & $\mathrm{gCO}_{2} / \mathrm{gBOD}$ & 0.33 \\
\hline$Y_{\mathrm{VSS}}$ & $\mathrm{gVSS} / \mathrm{gBOD}$ & 0.422 \\
\hline $\mathrm{r}_{\mathrm{O} 2}$ & $\mathrm{gO}_{2} / \mathrm{gBOD}$ & 0.4 \\
\hline \multicolumn{3}{|c|}{ Endogenose decay in aerobic process } \\
\hline $\mathrm{Y}_{\mathrm{CO} 2 \text {,decay }}$ & $\mathrm{gCO}_{2} / \mathrm{gVSS}$ & 1.56 \\
\hline $\mathrm{r}_{\mathrm{O} 2 \text {,decay }}$ & $\mathrm{gO}_{2} / \mathrm{gVSS}$ & 1.42 \\
\hline \multicolumn{3}{|c|}{ Anaerobic process } \\
\hline $\mathrm{Y}^{\mathrm{an}}{ }_{\mathrm{CO} 2}$ & $\mathrm{gCO}_{2} / \mathrm{gBOD}$ & 0.28 \\
\hline $\mathrm{Y}^{\mathrm{an}}{ }_{\mathrm{CH} 4}$ & $\mathrm{gCH}_{4} / \mathrm{gBOD}$ & 0.235 \\
\hline $\mathrm{Y}^{\mathrm{an}} \mathrm{VSS}$ & gVSS/gBOD & 0.035 \\
\hline \multicolumn{3}{|c|}{ Endogenose decay in aerobic process } \\
\hline $\mathrm{Y}^{\mathrm{an}} \mathrm{CO} 2$ decay & $\mathrm{gCO}_{2} / \mathrm{gVSS}$ & 0.58 \\
\hline $\mathrm{Y}_{\mathrm{CH} 4 \text {,decay }}^{\mathrm{an}}$ & $\mathrm{gCH}_{4} / \mathrm{gVSS}$ & 0.35 \\
\hline \multicolumn{3}{|c|}{ Nitrification process } \\
\hline$Y_{\mathrm{VSS}, \text { nit }}$ & $\mathrm{gVSS} / \mathrm{gN}$ & 0.55 \\
\hline $\mathrm{Y}_{\mathrm{NO} 3 \text {,nit }}$ & $\mathrm{gN}-\mathrm{NO}_{3} / \mathrm{gN}$ & 0.98 \\
\hline $\mathrm{r}_{\mathrm{O} 2 \text {,nit }}$ & $\mathrm{gO}_{2} / \mathrm{gN}$ & 4.32 \\
\hline $\mathrm{r}_{\mathrm{CO} 2 \text {,nit }}$ & $\mathrm{gCO}_{2} / \mathrm{gN}$ & 0.247 \\
\hline \multicolumn{3}{|c|}{ Denitrification process } \\
\hline$Y_{\mathrm{VSS}, \mathrm{dnt}}$ & $\mathrm{gVSS} / \mathrm{gN}-\mathrm{NO}_{3}$ & 0.175 \\
\hline $\mathrm{Y}_{\mathrm{CO} 2, \mathrm{dnt}}$ & $\mathrm{gCO}_{2} / \mathrm{gN}-\mathrm{NO}_{3}$ & 2.767 \\
\hline $\mathrm{r}_{\mathrm{BOD}, \mathrm{dnt}}$ & $\mathrm{gBOD} / \mathrm{gN}-\mathrm{NO}_{3}$ & 2.059 \\
\hline $\mathrm{r}_{\text {methanol,dnt }}$ & $\mathrm{gCH}_{3} \mathrm{OH} / \mathrm{gN}-\mathrm{NO}_{3}$ & 1.9 \\
\hline \multicolumn{3}{|c|}{ Anaerobic decay } \\
\hline $\mathrm{Y}_{\mathrm{CO} 2, \mathrm{dr}}$ & $\mathrm{gCO}_{2} / \mathrm{gBOD}$ & 0.28 \\
\hline $\mathrm{Y}_{\mathrm{CH} 4, \mathrm{dr}}$ & $\mathrm{gCH}_{4} / \mathrm{gBOD}$ & 0.23 \\
\hline$Y_{\mathrm{VSS}, \mathrm{dr}}$ & $\mathrm{gVSS} / \mathrm{gBOD}$ & 0.042 \\
\hline \multicolumn{3}{|c|}{ Biomass decay } \\
\hline $\mathrm{Y}^{\mathrm{dr}}{ }_{\mathrm{CO} 2 \text {,decay }}$ & $\mathrm{gCO}_{2} / \mathrm{gVSS}$ & 0.58 \\
\hline $\mathrm{Y}^{\mathrm{dr}}{ }_{\mathrm{CH} 4 \text {,decay }}$ & $\mathrm{gCH}_{4} / \mathrm{gVSS}$ & 0.35 \\
\hline
\end{tabular}

From the result of reactions, the coefficients of GHG emission are established as follows:

\section{Table 4: The coefficients of GHG emissions}

\section{Results and discussions}

\subsection{Operational process of No. 0 MTH model}

The No.0 MTH model was written by programing Scalable language. Input parameters of the model are: the quantity of electricity used, the quantity of material used, the quantity of material used and treatment capacity, BOD, TSS, TN, temperature of tanks, hydraulic retention time (HRT), sludge retention time (SRT), underflow rate, etc. Through the processes of calculating in the model will be obtained output results, which are off-site and on-site greenhouse gas emissions from the domestic wastewater treatment system. The model operating procedures are described in Table 5 and Figure 2 below:

\section{Table 5. Operational process of No. O MTH model}

\begin{tabular}{ll} 
Description & \multicolumn{1}{c}{$\begin{array}{c}\text { Operational process of No. O MTH } \\
\text { Model }\end{array}$} \\
\hline Inputs & - The off-site greenhouse gases emis- \\
& sions: \\
& + The quantity of electricity used \\
& + The quantity of natural gas used \\
& + The quantity of material used \\
& - The on-site greenhouse gases emis- \\
& sions: \\
& +Treatment capacity; BOD, TSS, TN \\
& +Temperature of tanks \\
& +Hydraulic retention time (HRT) \\
& +Sludge retention time (SRT) \\
& +Underflow rate \\
\hline Calculation & $\begin{array}{l}\text { Equations for calculation of the off-site } \\
\text { groenhouse gases emissions }\end{array}$ \\
& Equations for calculation of the on-site \\
& greenhouse gases emissions \\
\hline Outputs & $\begin{array}{l}\text { Emission results of the off-site green- } \\
\text { house gases }\end{array}$ \\
& Emission results of the on-site green- \\
& house gases \\
& Total emission results of the greenhouse \\
& gases
\end{tabular}

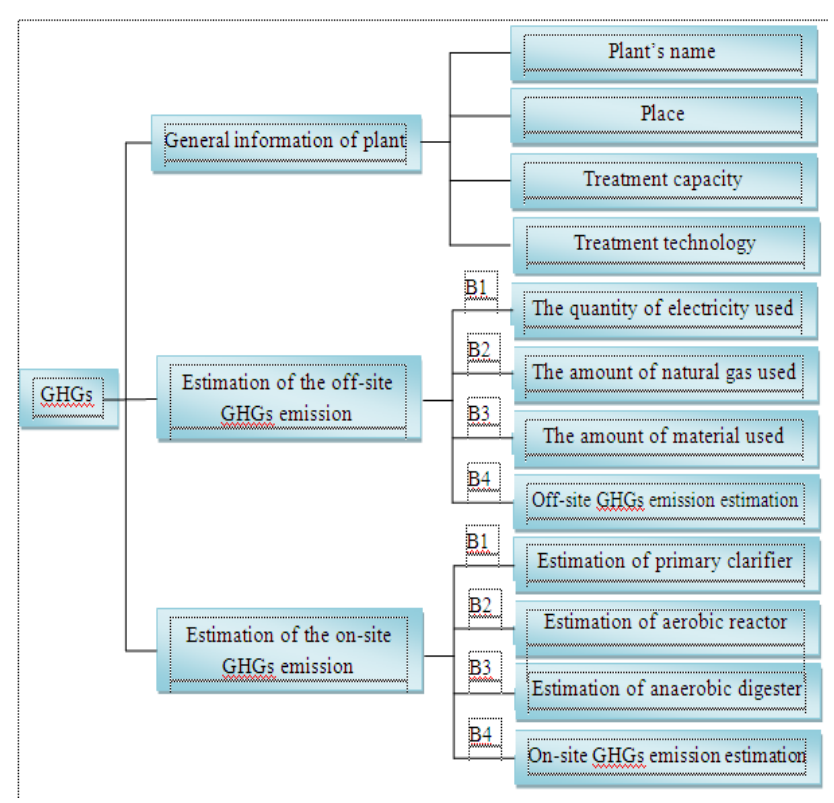

Figure 2. Flowchart of calculating greenhouse gas emissions from domestic wastewater treatment systems by No.0 MTH model

\subsection{Research results}

\subsubsection{Estimation of the off-site greenhouse gases emis- sion}

The No.0 MTH model was applied to calculate the greenhouse gas emissions from Yen So domestic wastewater 
treatment in HaNoi, with a treatment capacity of 120,000 $\mathrm{m}^{3} /$ day. Input parameters of the Yen So Plant and the results of calculation of greenhouse gas emissions indirectly were shown in table 6 and table 7. Through the No.0 MTH model simulated off-site greenhouse gas emissions from Yen So domestic wastewater treatment plants in Hanoi was $29,560.70 \mathrm{kgCO}_{2}$-eq / day.

Table 6. Inputs of the Yen So domestic wastewater treatment plant, Ha Noi

\begin{tabular}{lcc}
\multicolumn{1}{c}{ Parameter } & Unit & Value \\
\hline $\begin{array}{l}\text { Treatment capacity of } \\
\text { plant }\end{array}$ & $\mathrm{m}^{3} /$ day & 120,000 \\
$\begin{array}{l}\text { The quantity of electric- } \\
\text { ity used }\end{array}$ & $\mathrm{Kwh} /$ day & 26,400 \\
$\begin{array}{l}\text { The quantity of natural } \\
\text { gas used }\end{array}$ & $\mathrm{m}^{3} /$ day & - \\
$\begin{array}{l}\text { The quantity of material } \\
\text { used }\end{array}$ & & - \\
+ Alkalinity & $\mathrm{Kg} /$ day & 1,800 \\
+ PAC & $\mathrm{Kg} /$ day & 6,000 \\
+ Polimer & $\mathrm{Kg} /$ day & 180
\end{tabular}

Table 7. Outputs of the off-site greenhouse gases emission of the Yen So wastewater treatment plant, Ha Noi

\begin{tabular}{ccc} 
Result & Unit & Estimation value \\
\hline $\mathrm{P}_{\mathrm{CO} \text {, electricity }}$ & $\mathrm{kgCO}_{2 \text {-eq }} /$ day & $9,891.50$ \\
$\mathrm{P}_{\mathrm{CO} \text {, natual gas }}$ & $\mathrm{kgCO}_{2 \text {-eq }} /$ day & - \\
$\mathrm{P}_{\mathrm{CO} 2 \text {, material }}$ & $\mathrm{kgCO}_{\text {2-eq }} /$ day & $19,669.20$ \\
$\mathrm{P}_{\mathrm{CO} \text {, off-site }}$ & $\mathrm{kgCO}_{\text {2-eq }} /$ day & $29,560.70$ \\
$\mathrm{P}_{\mathrm{CO} 2 \text {, off-site }}$ & ton $\mathrm{CO}_{2 \text {-eq }} / \mathrm{year}$ & $10,789.60$
\end{tabular}

3.2.2. Estimation of the on-site greenhouse gases emission

The Yen So, Hanoi Plant treated domestic wastewater treatment by aerobic biological methods, combined with nitrogen removal and decomposition of biological sludge using anaerobic methods. Table 8 showed the kinetic parameters in during aerobic treatment, Nitrification and denitrification processes and anaerobic sludge. Table 9 showed the input parameters of the plant. Using No.0 MTH model calculated on-site greenhouse gas emissions from the factory Yen, Hanoi was $13,534.32 \mathrm{kgCO} 2$-eq / day and Emission factor was $2.506 \mathrm{kgCO}_{2-\mathrm{eq}} / \mathrm{kg} \mathrm{BOD}$ and shown in Table 10 .

Table 8. Kinetic parameters of the domestic wastewater treatment processes

a. Aerobic process

\begin{tabular}{lccc} 
Parameter & Unit & $\begin{array}{c}\text { Value at } 20^{\circ} \mathrm{C} \\
\left(\mathrm{K}_{20}\right)\end{array}$ & $\begin{array}{c}\text { Value at } \\
\left.22^{\circ} \mathrm{C} \mathrm{K}_{\mathrm{T}}{ }^{*}\right)\end{array}$ \\
\hline$\mu_{\mathrm{m}}$ & $1 /$ day & 4 & 4.58 \\
$\mathrm{Y}$ & $\mathrm{mg} / \mathrm{mg}$ & 0.50 & 0.60 \\
$\mathrm{k}=\mu_{\mathrm{m}} / \mathrm{Y}$ & & 8 & 7.63
\end{tabular}

\begin{tabular}{lccc} 
Parameter & Unit & $\begin{array}{c}\text { Value at } 20^{\circ} \mathrm{C} \\
\left(\mathrm{K}_{20}\right)\end{array}$ & $\begin{array}{c}\text { Value at } \\
\left.22^{\circ} \mathrm{C} \mathrm{K}_{\mathrm{T}}{ }^{*}\right)\end{array}$ \\
\hline $\mathrm{Ks}$ & $\mathrm{mg} / \mathrm{l}$ & 60 & 60 \\
$\mathrm{k}_{\mathrm{d}}$ & $1 /$ day & 0.10 & 0.11 \\
$\mathrm{f}_{\mathrm{d}}$ & & 0.1 & 0.10 \\
$(*) \mathrm{K}_{\mathrm{T}}=\mathrm{K}_{20} * \theta^{(\mathrm{T}-20)}$ & &
\end{tabular}

b. Nitrification and denitrification processes

\begin{tabular}{llcc} 
Parameter & Unit & Value at $20^{\circ} \mathrm{C}$ & Value at $22^{\circ} \mathrm{C}$ \\
\hline$\mu_{\mathrm{m}, \text { nit }}$ & $1 /$ day & 0.7 & 0.89 \\
$\mathrm{Y}_{\text {nit }}$ & $\mathrm{mg} / \mathrm{m}$ & 0.12 & 0.16 \\
$\mathrm{k}=\mu_{\mathrm{m}} / \mathrm{Y}$ & $\mathrm{g}$ & 7.50 & 5.58 \\
$\mathrm{~K}_{\mathrm{N}}$ & $\mathrm{mg} / 1$ & 0.5 & 0.87 \\
$\mathrm{k}_{\mathrm{d}, \text { nit }}$ & $1 /$ day & 0.08 & 0.04 \\
$\mathrm{~K}_{\mathrm{DO}}$ & & 1.3 & 1.3
\end{tabular}

c. Anaerobic process

\begin{tabular}{lcc} 
Parameter & Unit & Value at $30^{\circ} \mathrm{C}$ \\
\hline$\mu_{\mathrm{m}}{ }^{\mathrm{dr}}$ & $1 /$ day & 0.26 \\
$\mathrm{Y}^{\mathrm{dr}}$ & $\mathrm{mg} / \mathrm{mg}$ & 0.08 \\
$\mathrm{k}^{\mathrm{dr}}=\mu_{\mathrm{m}}{ }^{\mathrm{dr}} / \mathrm{Y}^{\mathrm{dr}}$ & $1 /$ day & 3.3 \\
$\mathrm{~K}_{\mathrm{s}}{ }^{\mathrm{dr}}$ & $\mathrm{mg} / \mathrm{l}$ & 380 \\
$\mathrm{k}_{\mathrm{d}}{ }^{\mathrm{dr}}$ & $1 /$ day & 0.03 \\
$\mathrm{f}_{\mathrm{d}}{ }^{\mathrm{dr}}$ & & 0.15
\end{tabular}

Table 9. Input parameters of the Yen So wastewater treatment plant, Ha Noi

\begin{tabular}{lccc} 
& Parameter & Unit & Value \\
\hline \multirow{4}{*}{ Inputs } & $\mathrm{Q}_{\mathrm{o}, \mathrm{v}}$ & $\mathrm{m}^{3} /$ day & 120,000 \\
& $\mathrm{~S}_{\mathrm{o}, \mathrm{v}}$ & $\mathrm{mg} / \mathrm{l}$ & 45 \\
& $\mathrm{TKN}_{\mathrm{v}}$ & $\mathrm{mg} / \mathrm{l}$ & 34 \\
& $\mathrm{TSS}$ & $\mathrm{mg} / \mathrm{l}$ & 51 \\
Primary & $\mathrm{Pr}_{\mathrm{bl}, \mathrm{BOD}}$ & $\%$ & 30 \\
clarifier & $\mathrm{Pr}_{\mathrm{bl}, \mathrm{SS}}$ & $\%$ & 40 \\
& $\mathrm{Q}_{\mathrm{bl}}$ & $\mathrm{m}^{3} /$ day & - \\
Aerobic & $\mathrm{Temperature}^{\circ}$ & ${ }^{\circ} \mathrm{C}$ & 22 \\
reactor & $\mathrm{SRT}$ & day & 5 \\
& $\mathrm{HRT}$ & hour & 5 \\
Anaerobic & $\mathrm{Temperature}$ & ${ }^{\circ} \mathrm{C}$ & 30 \\
digester & $\mathrm{SRT}$ & day & 20
\end{tabular}

Table 10. Output parameters of the on-site greenhouse gases emission of the Yen So wastewater treatment plant, Ha Noi

\begin{tabular}{lcc} 
Result & Unit & $\begin{array}{c}\text { Estimation va- } \\
\text { lue }\end{array}$ \\
\hline Aerobic reactor & & \\
$\mathrm{CO}_{2, \text { aerobic reactor }}$ & $\mathrm{kg} /$ day & $8,539.09$ \\
$\mathrm{CO}_{2, \mathrm{~N} 2 \mathrm{O} \text { emission }}$ & $\mathrm{kg} /$ day & $4,830.72$ \\
Anaerobic digester & &
\end{tabular}




$\begin{array}{lcc}\mathrm{CO}_{2, \text { anaerobic digester }} & \mathrm{kg} / \text { day } & 17.29 \\ \mathrm{CO}_{2 \text { equivalent }} & \mathrm{kg} / \text { day } & 147.22 \\ \text { Total on-site } & \mathrm{kg} / \text { day } & 13,534.32 \\ \text { GHGs emission } & & \\ \text { Total on-site } & \text { tons/day } & 4,490.03 \\ \text { GHGs emission } & & 2.506 \\ \text { Emission factor } & \mathrm{kgCO}_{2 \text {-eq }} / \mathrm{kgBOD} & 2.06\end{array}$

\section{Conclusions}

In this research, the No.0 MTH model was applied for estimating the greenhouse gas emission from domestic wastewater treatment systems. The model was written by programing Scalable language and model formulations were based on equations of substrate and biomass, biochemical reactions and Monod kinetics equations for biological treatment reactors.

The No.0 MTH model was applied in calculating the greenhouse gas emissions of Yen So domestic wastewater treatment plant in HaNoi. Calculation results showed that amount of off-site greenhouse gas emissions 2 times higher than the amount of on-site greenhouse gas emissions and on-site greenhouse gas emission factor as $2,506 \mathrm{kgCO}_{2 \text {-eq }} /$ kgBOD.

The No.0 MTH model can be applied to calculate the greenhouse gas emissions from domestic wastewater treatment systems with similar treatment method.

With variation of input parameters in the model, through the processes of calculating the model, will simulate the results of greenhouse gas emissions from domestic wastewater treatment systems. From these results, the operational and technological parameters could be chosen to reduce the greenhouse gas emission from these domestic wastewater treatment systems.

\section{References}

[1] Annual report of EVN, 2012 - 2013

[2] Bani Shahabadi M., Yerushalmi L., Haghighat F. (2009) Impact of process design on greenhouse gas (GHG) generation by wastewater treatment plants. Water Research 43:2679-2687O. Ashrafi, L. Yerushalmi, F. Haghighat. (2013). Mathematical modeling of GHG emission in wastewater treatment plants: steady-state vs. dynamic. CCTC 2013 Paper Number 1569701483

[3] Rashad S.M., Hammad F.H. (2000) Nuclear power and the environment: Comparative assessment of environmental and health impacts of electricity-generating systems, The 7th International Energy Conference (ENERGEX '98), November 19, 1998 - November 21, 1998, Elsevier Ltd, Isa Town, Bahrain. pp. 211229.

[4] The 2006 IPCC Guidelines for National Greenhouse Gas Inventories (IPCC,2006).

[5] The report reviews the management of urban waste water in Vietnam, World Bank, 2013.

[6] Urban sewage treatment, Tran Duc Ha, 2006 